\title{
BRAINWAVE ASSISTIVE SYSTEM FOR PARALYZED INDIVIDUALS
}

\author{
Md Ahnaf Shariar ${ }^{1}$, Syeda Maliha Monowara², Md. Shafayat Ul Islam³, \\ Muhammed Junaid Noor Jawad ${ }^{4}$, Saifur Rahman Sabuj ${ }^{5}$ \\ 1,2,3,4 Department of Electrical and Electronic Engineering, BRAC University, Bangladesh, ${ }^{5}$ Department of Electronics and \\ Control Engineering, Hanbat National University, South Korea
}

NOTE: Corresponding author: Md Ahnaf Shariar, md.ahnaf.shariar@g.bracu.ac.bd

\begin{abstract}
The Brain-Computer Interface (BCI) is a system based on brainwaves that can be used to translate and comprehend the innumerable activities of the brain. Brainwave refers to the bioelectric impulses invariably produced in the human brain during neurotransmission, often measured as the action potential. Moreover, BCI essentially uses the widely studied Electroencephalography (EEG) technique to capture brainwave data. Paralysis generally occurs when there is a disturbance in the central nervous system prompted by a neurodegenerative or unforeseen event. To overcome the obstacles associated with paralysis, this paper on the brainwave-assistive system is based on the BCI incorporated with Internet-ofthings. BCI can be implemented to achieve control over external devices and applications. For instance, the process of cursor control, motor control, neuroprosthetics and wheelchair control, etc. In this paper, the OpenBCI Cyton-biosensing board has been used for the collection of the EEG data. The accumulated EEG data is executed subsequently to obtain control over the respective systems in real-time. Hence, it can be concluded that the experiments of the paper support the idea of controlling an interfaced system through the real-time application of EEG data.
\end{abstract}

Keywords - Action potential, brain-computer interface, brain wave, central nervous system, electroencephalography data

\section{INTRODUCTION}

The Brain-Computer Interface (BCI) has articulated a phenomenon that bridges the neural activities in the human brain with the computer system. The study of brainwave oscillations has been conducted by pioneers since the early 1900s. However, the research on BCI technology progresses towards non-traditional advancement in recent times due to the availability of wireless means of communication [1]. Nonetheless, the emerging research on implantation for interfacing with the neural system in [2], suggests an enhancement in the brainwave data acquisition process. In hindsight, the asserted techniques of accumulating data through conventional invasive methods can be substituted by biomedical treatments as discussed in [3]. The application of the Internet of Bio-Nano Things (IoBNT), expedited the implantation of the electrodes of artificial neurons to provide state-of-the-art technological facilities. Besides, [4] proposes how a nanogenerated neural network can provide improved stimulation of brainwave signals for an optimized control system. Nevertheless, the high risk of tissue damage might considerably precede the favorable features of direct stimulation of prosthesis, muscles, and the spinal cord [3]. Provided the contingency factors, the non-invasive approaches have greater possibilities of being embraced, especially in the middle-income countries around the world.

The brainwave impulses generated during neurotransmission in the Central Nervous System (CNS) can be computed and interpreted using the $\mathrm{BCI}$ convention. Thus, the plausibility of the system is inherently conditioned to the user's brain functionality $[5,6]$. BCI essentially uses the Electroencephalography (EEG) technique for the retrieval of the aforementioned signals, in the form of frequencies and electric bio-signals [7]. Recent studies suggest that the brain's peripheral mechanism for motor functionalities is not as imperative as anticipated over the years. Generally, the EEG data produced in a paralyzed individual is indistinguishable from that of a non-paralyzed person. Therefore, BCI can be employed to obtain Neurofeedback (NFB) of the complex brain activities by restoring the envisioned stimulation [8]. Consequently, successful task completion can be facilitated by utilizing brainwave signals alone, without requiring any physical actions. Hence, it can considerably assist paralyzed and physically 
impaired individuals to regain self-sufficiency through technological breakthroughs; it can also benefit the field of clinical research and studies of neuroscience applications.

Furthermore, the collected ranges of data have boundless means of implementation if executed diligently. In [9] the primary focus has been the interpretation of the correlation between the brainwave activities while playing various computer games. The fundamental aspect of a different study [10] utilizes the Steady-State Visual Evoked Potential (SSVEP) based BCI system. Whereupon, the control over an automated wheelchair has been executed as a response to visual stimulation. On the other hand, [11] analyses the composition of music in correspondence to human feelings by employing the user interface of the BCI system. In our paper, the OpenBCI Cyton board has been operated as the EEG-based device for brainwave activity determination. Generally, such devices have been extensively used due to their advantageous factors of real-time responses, portability, and affordability [12]. Moreover, the techniques applied for data acquisition in our paper, and the respective ones in $[10,14,19]$ were comparable, regardless of the indication of the stimulus. For instance, [14] coordinated the visual functioning alongside the pedaling action of the user and interfaced with the system. On the other hand, $[10,19]$ have rather depended on the user's response to visual stimulation. On the other hand, [18] has suggested a study for improvement in stroke patients by implementing the composed brainwave data.

Nevertheless, in this paper, we have accumulated the non-invasive EEG readings by placing electrode cups throughout the surface area of the scalp. The corresponding non-intersecting brainwaves exhibited are commonly classified as delta, theta, alpha, beta and, gamma frequencies. The delta $(<4 \mathrm{~Hz})$ and theta $(4-7 \mathrm{~Hz})$ frequencies are observed in a relatively calmer state such as in the deep-sleep or relaxed state. Alpha (8-12 Hz) waves are commonly related to passive awareness and perception, they are generally discovered in the optical region of the brain. The beta $(12-30 \mathrm{~Hz})$ and gamma (30-100 $\mathrm{Hz})$ waves are witnessed in the higher ranges due to the association with attention, cognition, and motor functionalities, related to the central and frontal lobes of the brain [12]. For our paper, the beta, and gamma ranges of frequencies have been exclusively centred for the implementation of cognitive functioning and control-based operations. Wireless networking methodology enables the successful communication of the EEG readings with the computer system. The OpenBCI Wi-Fi shield and RFDuino are both means of wireless communication. However, we have interfaced the Wi-Fi shield to transmit the gathered data through the medium of the Internet.

\section{MOTIVATION AND CONTRIBUTION}

In this paper, the proposed methodology would inherently enable the paralyzed people to overcome most of the technological barriers. As per the World Health Organization, a staggering 20-50 million individuals suffer from some degree of physical impediments caused by injuries and car accidents [6]. More often than not, the victims of paralysis and physical disabilities exhibit the immaculate generation of neuro-electrical impulses identical to that of a healthy brain. This implies that the brainwave signals obtained from the EEG data of an individual can be configured to be transmitted as input commands to retain control over a designated system. In order to ensure the performance of the system, we first scrutinized the numerous prospects of the EEG data obtained through the BCI technology. The continuous stream of data documented the ceaseless activity of brainwaves in an individual. However, the distinct functionalities of the brainwave at certain instances signified the constricted ranges of the collected data. Therefore, the perceptive administration in the cognitive frequency ranges has allowed attaining technological control in real-time. Our primary objective has been to enable physically impaired individuals to subdue certain limitations through the implementation of the BCI system. Moreover, the secondary objective is to utilize the invoked potential from the user's brain activity for different real-life implementations similar to that of [10]. Unlike the tactile operating system demonstrated in [16], which depends on an external control unitour goal is to achieve command through the implementation of brainwave signals only.

Our contributions in this paper are as follows:

- To investigate the plausibility of the incorporation of the accumulated data to gain control over the proposed system.

- To discuss the possibility of the findings through the real-time implementation of the EEG data in an exploratory approach. 
The paper holds the anticipation of providing ready-to-use technology without any modification. Although, the proposed system is yet to undergo multiple further steps of trial and error before it can be actively used. Nonetheless, the model has been partially established in order to experimentally incorporate the completion of specific tasks. In addition to that, there are challenges of managing and operating the innumerable brainwave data that is being constantly collected. Furthermore, the constricted period of data processing might challenge the efficiency and complexity during the application, which also needs to be considered. The proposed methodology of the system of our paper has been discussed elaborately in Section 3. This is succeeded by, the experimental feedback generation is presented in Section 4. Lastly, results and discussion are provided in Section 5.

\section{PROPOSED METHODOLOGY}

The proposed methodology section intricately represents the intended system architecture of our paper, as illustrated in Fig. 1. To ensure ample competency of the system, the pathway consists of data acquisition, data processing and data implementation. The data acquisition segment focuses on the processes of receiving the EEG signals from the brain. Hence, the accumulated data from the subject emphasizes optimizing its applications by eliminating any and/or all initial errors. Upon the collection of the data, the Wi-Fi shield instantly allows the transmission to a computer by means of wireless communication. The data processing further accumulates data in the next phase, whereby it is processed in order to filter and minimize external signals, noise, jitters, etc. Thus, it ensures that the acquired data can be further processed or preserved for future analysis and applications. Moreover, the data can be configured to be globally accessible at any instance through the wireless communication of $5 \mathrm{G}$ devices. Besides, the signals can also be transmitted to assigned wireless systems for the observation of the generated NFB. Eventually, the data implementation section illustrates how we have experimentally implemented the data through feedback generation of the control-specific data. In our paper, two dissimilar approaches have been observed for the experimental feedback of the control applications. The detailed discussion has been mainly segmented into the three aforementioned units which have been associated with each other and described henceforth:

\subsection{Data acquisition}

The fundamental component employed in our paper is the OpenBCI Cyton board. The board uses the EEG technique to extract the data from the brain's neural oscillations [17]. The neural impulses are initially analyzed as Action Potentials (AP) that are commonly measured as microvolts. The illustration of the AP, also known as biopotential of the brainwave during various activities has been compared in association to [17]. The 8-channels of the board have 8 individual electrodes connected to them, which are used for the collection of the EEG data. The electrode goldcups are placed on the scalp of the user according to the 10-20 arrangement as observed in $[10,19]$, this assures the accuracy of the obtained brainwave data. Moreover, to guarantee the precise transmission of the data, conductive pastes are applied along with the electrodes. We have accumulated the featured extraction of brainwave signals accordingly. Furthermore, in our paper, a WiFi-shield has been connected along with the Cyton board which allows signal transmission and networking. Therefore, the visualization of the signals in different instances and domains can be possible through the interface with suitable applications.

However, unlike the pedaling technique of brainwave stimulation associated in [14], our approach required little to no movement but only focus control. To ensure the elimination of the motor functionalities, the experiments conducted in our paper concentrated on the cognitive and visual aspects of the user. The number of 


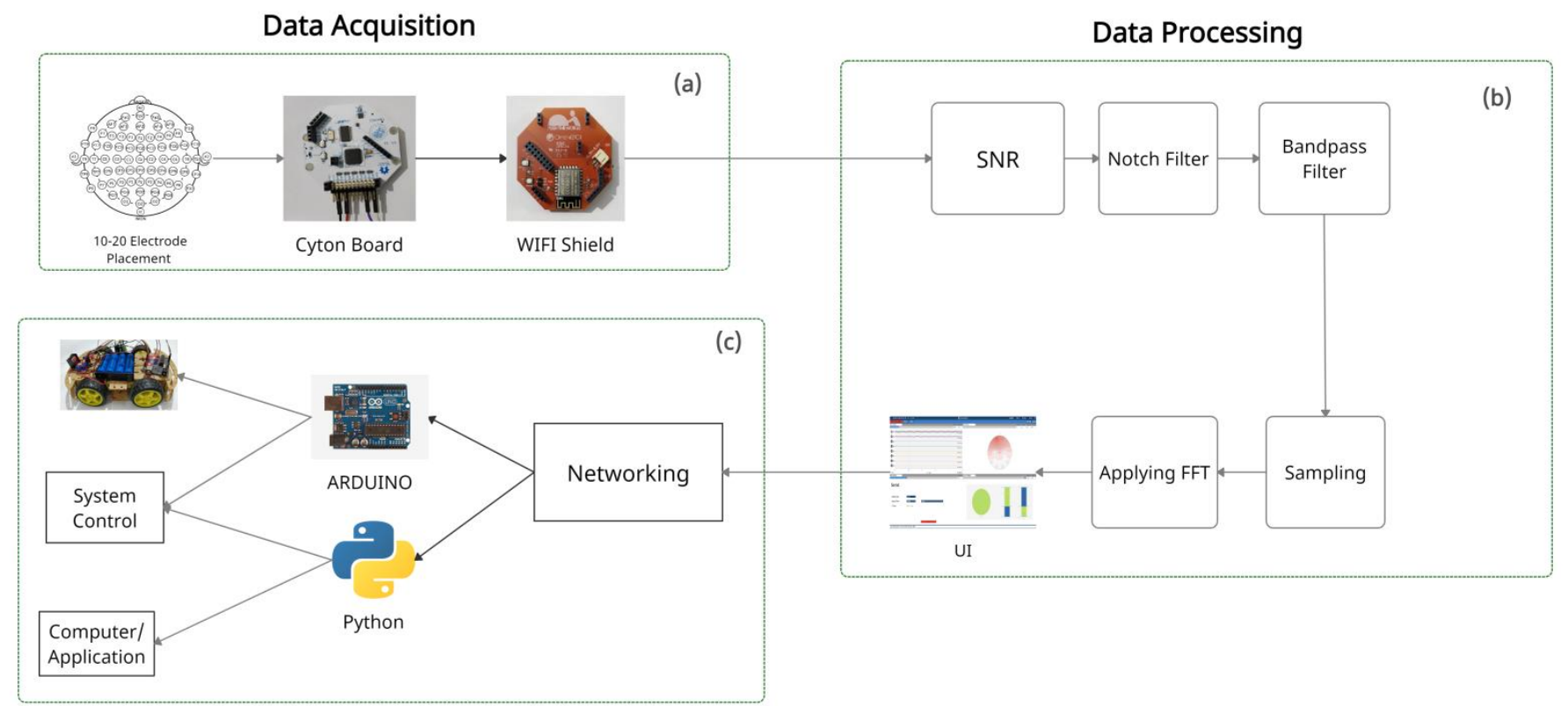

Data Implementation

Fig. 1 - System architecture [20]

experiments we have administered thus faressentially monitors the variations in EEG data during the motion of the arm or eye. It aspires to justify the objective of our paper by comparing the focus state and cognition to the developments in physical movement. The experiments directed in [21] have a comparable approach to that of ours. However, the control of an exoskeleton requires an expanded range of focus-control mechanisms which employs a considerable number of electrodes. In [21], the connectivity of the extensive driver circuits is maintained by the 16-electrodes channels that provide higher accuracy of brainwave data.

As discussed earlier, the inherent performance of the brain associated with the intellectual and comprehensive features remains consistent regardless of the capability of muscle control [6]. Although the subject of our paper exhibits the functionality of a healthy brain, the constrained ranges of the accumulated EEG data has facilitated the execution of the applications as designed. Consequently, [6] administered the control of brainwave frequency in the ranges $0.15-5 \mathrm{~Hz}$ in the time-span of $300-600 \mathrm{~ms}$ by the commonly used P300 in the BCI system. On a similar account, $[10,14,17-19]$ represents the manipulation of the acquired brainwave data in the targeted bandwidth for the respective allocations.

\subsection{Data processing}

As per Fig. 1, the acquired brainwave data requires extensive steps of processing and optimization before it can be analyzed. Thus, the discussion in [10] embodies the construction of the anticipated data processing operations through the user interface. Our foremost concern is the noise interferences associated with the raw brainwave data. Ordinarily, the non-invasive method contributes to the factors of noise since brainwave frequencies are not being directly extracted. Natural obstacles existing between the electrodes and the brain, i.e. the skull, skin, hair, etc. as well as artefacts (muscular movements) contribute to the noises [19]. To optimize the data, the user interface applies a Signal-to-Noise Ratio (SNR) for strengthening the signal that is estimated by applying the root-mean-square $[10,18]$. Furthermore, signal filtering and sampling facilitate the selection of the desired ranges of data to be executed accordingly. The sampling theory of Nyquist-Shannon ensures that the accumulated EEG signals are sampled at a frequency of $250 \mathrm{~Hz}$ by the user interface applications [19]. Upon the passage of the frequencies through the Butterworth filters, the aspired ranges are separated for assigned requisitions. Additionally, the presence of electronic devices that emit electromagnetic waves of $50-60 \mathrm{~Hz}$ can cause distortion. Hence, to eliminate the specific bandwidth, notch filters are applied in the system. 
Moreover, the Fast Fourier Transform (FFT) approach allows the observation of the brainwaves in the frequency-domain corresponding to the AP or bio-potential readings $[10,14]$. Hence, this provides a more transparent comprehension of the acquired data when examined.

\subsection{Data implementation}

Henceforth, the accumulated data undergoes further networking for implementation purposes. For instance, a Lab Streaming Layer (LSL) networking protocol is employed to transmit the data from one application to another. Correspondingly, the optimized brainwave data in our paper has been transferred from the OpenBCI network to the Python application. This has permitted the user for visualization and controlled scrolling of newsfeed through EEG data. The communication between such applications yields the graphical representation of the brainwave AP in various domains as illustrated in [19].

In the case of interfaces with microcontrollers such as the Arduino, a serial communication protocol is exercised. The streaming of the brainwave signal in our paper has been enabled by the networking widget of the OpenBCI application. The successful serial communication has been sustained through the maintenance of the designated baud rate of EEG data streaming. Moreover, the transmission is implemented for NFB generation through the assigned receiving ports of the Arduino. As demonstrated in [10], the final implementation of controlling a wheelchair has been successfully achieved through the interfaced microcontroller. Therefore, it can be implemented for numerous other purposes such as robotics, neuroprosthetics, control of gadgets, appliances, and vehicles. Moreover, the use of diverse microcontrollers may as well accomplish related applications. For example, in [14] the pedaling bike was interfaced with the computer through the Raspberry Pi. Further discussion of the conducted experimental NFB generation in our paper is continued in the next section.

\section{EXPERIMENTAL FEEDBACK GENERATION}

\subsection{Hardware implementation}

The assertion of the earlier experiments depends on the support of the outcomes of the feedback generation observed. As orchestrated in Fig. 2, the Arduino relies on the serial communication networking protocol. In our paper, the NFB of EEG data was delivered through hardware implementation of the Arduino. Hence, it has allowed the accumulated data to be used for control and observation. Thus far, we have retained the motion of a small-scale car by utilizing the generated EEG feedback. The authority is maintained in the focus-state frequency ranges of the brainwave signals. Nonetheless, it is essentially a representation of the motion and direction control of a large-scale carrier, such as a wheelchair. In comparison to the electric wheelchair control in [10] and the bike pedaling in [14], where the visual stimulation required a cue/signal such as LED, stimulation as such was not imposed in our paper. Rather, the control of the car was perceived at the very instance the user accessed their focus-state. The user continued to operate the motion of the car upon the period of concentration and high cognitive functioning. It further enabled the user to control the direction of the car by propelling cognitive thought processes and absolutely no other means of administration. Eventually, the car immediately stopped when the focus state was departed.

\subsection{Software control observation}

Furthermore, the feedback generation of our paper was further observed through computer software applications. As illustrated in Fig. 2, LSL networking enables data transmission from the user interface to a Python application. Hence, the controlling of the newsfeed and computer screen has been conceivable. Similarly, in [5], cursor control was obtained by using the EEG data from the BCI. Suggesting that further precision in controlling virtual platforms can be achieved by enhancing goal-oriented or high-level programming language. For instance, the visual 
cortex of the occipital lobe can enable the user to spell out the visualized words and letters as shown in [15]. However, to deliver such precise commands in any system, deep learning of the data is imperative. As associated in [13], the machine learning algorithm of deep learning concentrates on the advancements of precision-control in automated systems. This can benefit the obtained feedback generation of our paper to incorporate concrete real-life applications in the future.

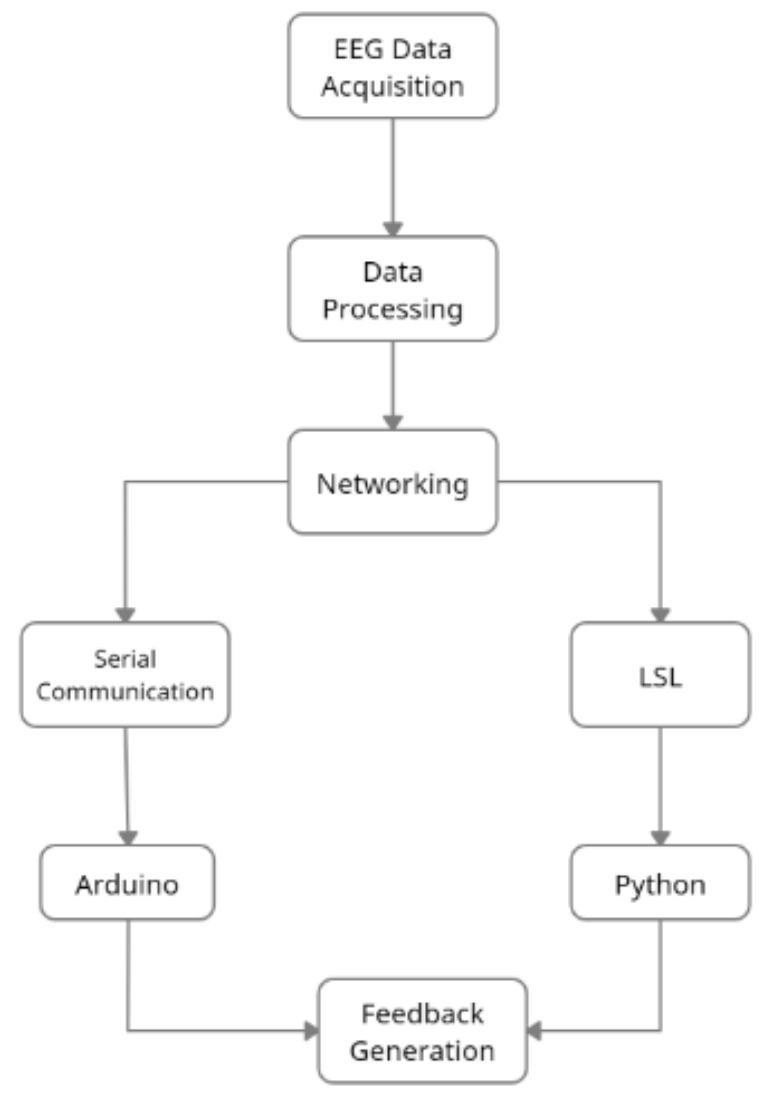

Fig. 2 - Experimental feedback generation

\subsection{Parameter settings}

In Section 4.1 and Section 4.2, hardware implementation and software control implementation were observed by utilizing the data streaming of an EEG reading from the person. Correspondingly, the EEG data generated from mostly the parameter setting depends on the pathway taken to filter the signal. Most of the time depending on the desired frequency range the filter setting changes to bandpass filter. For instance, to find out cognitive functioning EEG data, electrodes are attached to the frontal lobe, where to get rid of external electrical influence a notch filter is used. In addition, a bandpass filter of $5-50 \mathrm{~Hz}$ is used, which is the brain activity wave except for the delta which is experienced during meditation or dreamless sleep and the amount of sample rate varies depending on the outcome expectation but mostly the used sample rate is $255 \mathrm{~Hz}$ [18]. Moreover, maximum frequency can be opted for according to the preference which could be from $20 \mathrm{~Hz}$ to $400 \mathrm{~Hz}$. Again, to make the data transmitted to Arduino or Python workable mapping of the data is required. Moreover to that, mapped data is used in the completion of tasks or controlling a system.

\section{RESULT AND DISCUSSION}

Continuing on from the previous discussion, motion control of a car with the assistance of Arduino has been successfully implemented by utilizing the acquired brainwave data exclusively. The Arduino-based robotic car has been through multiple trials for motion control where the brain wave signals were used to enforce the focus and concentration state on a real-time basis [20]. Looking at Fig. 3, we can see that 3(a) displays the bio potential of the brainwave in the frequency domain while 3(b) and 3(c) display the head region activity at the instances. In Fig. 3(a), 2 channels were attached to the frontal lobe while acquiring concentration state data. In 3(a) the FFT plot of the potential values are majorly active in the alpha (8-12 Hz) and beta $(13-32 \mathrm{~Hz})$ frequency ranges. This information implies that the focus state is activated in the aforementioned bandwidths and the car gains motion correspondingly. Thus, the motion and direction of the car can be controlled with concentration. However, the car immediately stops moving the very moment the focus state is departed. Furthermore, the head region plots $3(\mathrm{~b})$ and 3(c), display high activity in the frontal and central lobe. The highlighted regions of the respective lobes are distinctly represented in the event of cognitive and motor functionalities. 


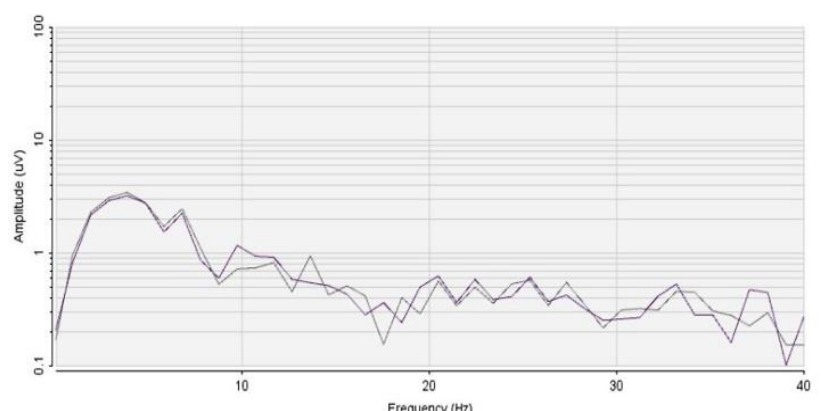

(a)

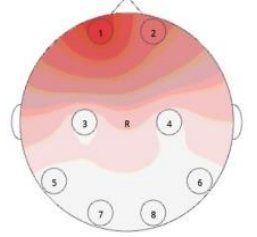

(b)

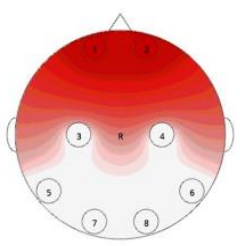

(c)
Experiment-1

Fig. 3 - Hardware implementation observation

Additionally, brainwave data has also been implemented in [20] for the control system of scrolling through computer applications. Thus, the user has been able to surf through a news feed or webpage while within the focus state as discussed earlier. In Fig. 4, 4(a) shows the FFT plot of the EEG data, 4(b) and 4(c) shows the head region plots during the monitoring and controlling of the virtual screen. For Fig. 4(a), on the head scalp near the occipital region one channel was attached to acquire the visualization data. As indicated in 4(a), the ranges of potential spikes are over a wider range of frequency. However, the head region plots display activity in the occipital region which wasn't as visible in the previous section. The mapping of the EEG data between the frontal and occipital lobe has enabled the opportunity to control the cursor by comparison of the action potentials. Further precision of the control can be enabled through deep learning techniques of the brainwave data.

The fundamental aspiration of the paper was to successfully acquire brainwave data with the assistance of the OpenBCI Cyton board in real-time. Upon the initial observation of the collected data, it emerged as a burst of reading due to the continuous brain activity. Since the brain is continuously generating signals, the data accumulation in the aspired range for application has been considerably challenging.
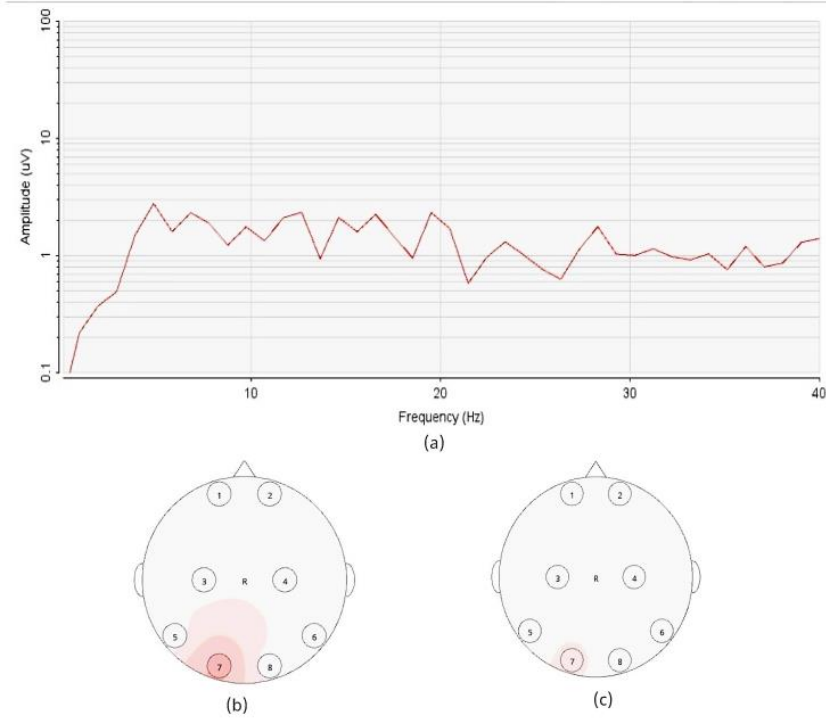

Experiment-2

Fig. 4 - Software control observation

Additionally, the noise factors are incorporated in the distortion of the quality of the raw data. The invariable muscular movements were incorporated as noise artefacts that required further extensive processing. Hence, the constraints over the potential values were challenging hindrances while attempting to accumulate the desired frequency ranges in order to implement certain requirements as per the respective cases.

Furthermore, the motion control of the car experiment suggested that the slightest deviation of the correspondence between the frontal and the parietal lobe can cause a significant disparity in the motion of the car. Similarly, the cursor control system may lack stability caused by equivalent factors. Hence, any inconsistency of focused cognitive functioning may disrupt the entire control system regardless of the system outcome. Nonetheless, our analysis establishes the fact that specific tasks can be performed up to a certain degree by utilizing EEG brainwave data. For further accuracy of the implementation of the feedback generation, more precision is required through machine learning approaches. 
Table 1 - Data table for cognitive functioning

\begin{tabular}{|l|l|}
\hline Channel 1 & Channel 2 \\
\hline-8762.24 & -1654.23 \\
\hline-8680.41 & -1641.42 \\
\hline-7665.33 & -2408.71 \\
\hline-7226.03 & -2746.78 \\
\hline-7854.65 & -2301.63 \\
\hline-8755.38 & -1620.7 \\
\hline-8709.45 & -1639.95 \\
\hline-7669.87 & -2445.26 \\
\hline-7241.63 & -2752.37 \\
\hline-7833.17 & -2260.28 \\
\hline-8733.85 & -1601.48 \\
\hline-8707.7 & -1643.84 \\
\hline-7682.54 & -2453.39 \\
\hline-7209.76 & -2752.51 \\
\hline-7824.81 & -2268.88 \\
\hline-8728.29 & -1647.03 \\
\hline-8722.86 & -1656.98 \\
\hline-7701 & -2421.3 \\
\hline-7238.48 & -2782.5 \\
\hline-7821.79 & -2314.9 \\
\hline-8732.8 & -1594.57 \\
\hline & \\
\hline
\end{tabular}

Table 1 illustrates the raw data of brainwave reading during cognitive functioning. The subject was asked to read and concentrate between the focus and out-of-focus state. During which, only the channels connected to the frontal region of the brain were kept activated. The collected data is transmitted to Arduino through means of serial communication, and thus, the motion of the car is controlled.

Furthermore, Table 2 demonstrates a portion of the data table from the acquired brainwave connected to a single channel in the occipital region of the brain. However, the tables provide the raw data that had been initially obtained. Henceforth, the use of Python and the lab streaming layer allowed the live streaming of the brainwave data, which was observed during the software control task.
Table 2 - Data table for occipital functioning

\begin{tabular}{|l|l|}
\hline Channel 7 & Channel 8 \\
\hline-45548 & 0 \\
\hline-45529.09 & 0 \\
\hline-45505.09 & 0 \\
\hline-45511.95 & 0 \\
\hline-45524.82 & 0 \\
\hline-45539.8 & 0 \\
\hline-45550.55 & 0 \\
\hline-45539.89 & 0 \\
\hline-45545.79 & 0 \\
\hline-45539.69 & 0 \\
\hline-45525.16 & 0 \\
\hline-45503.43 & 0 \\
\hline-45484.52 & 0 \\
\hline-45492.59 & 0 \\
\hline-45491.92 & 0 \\
\hline-45496.77 & 0 \\
\hline-45506.79 & 0 \\
\hline-45513.67 & 0 \\
\hline
\end{tabular}

\section{CONCLUSION}

The analysis of the brainwave signals developed over the decades has laid the foundation for the notion of the BCI-based control system. The possibilities of implementing BCI can have a profound impact on all branches of science and technology, creating opportunities for every individual regardless of their physical capabilities. The IoT based brainwave assistive system for paralyzed individuals is designed to attain opportunities as such. The results achieved thus far, delivers beneficial analysis and provides insights on further potential of the brainwave data. However, our future aspects of the paper envisions precision control of large-scale automated devices such as a wheelchair. Hence, for expanding accuracy control through further propagation of the data, machine learning propositions will be incorporated to the current paper. 


\section{ACKNOWLEDGEMENTS}

We would like to convey our heartfelt gratitude to the Department of Information and Communication Technology (ICT Division) of the Bangladesh Government, for their generous support as the sponsor of our paper.

\section{REFERENCES}

[1] F. Lotte, C. S. Nam, and A. Nijholt, "Introduction: Evolution of Brain-Computer Interfaces," Brain-Computer Interfaces Handbook: Technological and Theoretical Advance, Taylor \& Francis (CRC Press), pp. 1-11, 2018, doi: 9781498773430 〈hal-01656743〉.

[2] D. Accoto, M. T. Francomano, A. Rainer, M. Trombetta, P. M. Rossini, and E. Guglielmelli, "An implantable neural interface with electromagnetic stimulation capabilities," Medical Hypotheses, vol. 81, no. 2, pp. 322-327, May 2013, doi: https://doi.org/10.1016/j.mehy.2013.04.008.

[3] 0. B. Akan, H. Ramezani, M. Civas, 0. Cetinkaya, B. A. Bilgin, and N. A. Abbasi, "Information and Communication Theoretical Understanding and Treatment of Spinal Cord Injuries: State-of-the-art and Research Challenges," IEEE Reviews in Biomedical Engineering, Feb. 2021, doi: https://doi.org/10.1109/RBME.2021.3056455.

[4] S. Lee, H. Wang, Q. Shi, L. Dhakar, J. Wang, N. V. Thakor, S.-C. Yen, and C. Lee, "Development of battery-free neural interface and modulated control of tibialis anterior muscle via common peroneal nerve based on triboelectricnanogenerators (TENGs)," Nano Energy, vol. 33, pp. 1-11, Mar. 2017, doi: https://doi.org/10.1016/j.nanoen.2016.12.038.

[5] X. Gu, Z. Cao, A. Jolfaei, P. Xu, D. Wu, T.-P. Jung, and C.-T. Lin, "EEG-based Brain-Computer Interfaces (BCIs): A Survey of Recent Studies on Signal Sensing Technologies and Computational Intelligence Approaches and their Applications," IEEE/ACM Transactions on Computational Biology and Bioinformatics, Jan. 2020, doi: arXiv:2001.11337.
[6] A. Raj and S. M. Jaisakthi, "Analysis of Brain Wave Due to Stimulus Using EEG," 2018 International Conference on Computer, Communication, and Signal Processing (ICCCSP), Chennai, 2018, doi: 10.1109/icccsp.2018.8452823.

[7] K. Uno, G. Naito, Y. Tobisa, L. Yoshida,Y. Ogawa, K. Kotani and Y. Jimbo, "Basic Investigation of Brain-Computer Interface Combined with Augmented Reality and Development of an Improvement Method Using the Nontarget Object," Electronics and Communications in Japan, vol. 98, no. 8, pp. 9-15, Jul. 2015, doi: 10.1002/ecj.11715.

[8] S. Kono and T. M. Rutkowski, "Tactile-force brain-computer interface paradigm Somatosensory multimedia neurotechnology application," Multimedia Tools and Applications, vol. 74, pp. 8655-8667, Oct. 2015, doi: 10.1007/s11042-014-2351-1.

[9] A. G. Bakaoukas, F. Coada, and F. Liarokapis, "Examining brain activity while playing computer games," J Multimodal User Interfaces, vol. 10, pp. 13-29, 2016, doi: https://doi.org/10.1007/s12193-0150205-4.

[10] Y. Li, K. K. Ang, and C. Guan, "Digital Signal Processing and Machine Learning," BrainComputer Interfaces, The Frontiers Collection, Springer, Berlin Heidelberg, pp. 305-330, Sep. 2010, doi: 10.1007/978-3642-02091-9_17.

[11] M. Numao, T. Nishikawa, T. Sugimoto, S. Kurihara, and R. Legaspi, "Constructive Adaptive User Interfaces Based on Brain Waves," Human-Computer Interaction, Springer-Verlag Berlin Heidelberg, vol. Part II, no. HCII 2009, pp. 596-605, 2009.

[12] Vaibhaw, J. Sarraf, and P. K. Pattnaik, "Braincomputer interfaces and their applications," An Industrial IoT Approach for Pharmaceutical Industry Growth, vol. 2, pp. 31-54, 2020, doi: https://doi.org/10.1016/B978-0-12821326-1.00002-4.

[13] M. Rashid, N. Sulaiman, A. P. P. Abdul Majeed, R. M. Musa, A. F. Ab. Nasir, B. S. Bari, and S. Khatun, "Current Status, Challenges, and Possible Solutions of EEG-Based BrainComputer Interface: A Comprehensive Review," Frontiers in Neurorobotics, Jun. 2020, doi: 10.3389/fnbot.2020.00025. 
[14] M. A. Romero-Laiseca, D. Delisle-Rodriguez, V. Cardoso, D. Gurve, F. Loterio, J. H. PossesNascimento, S. Krishnan, A. FrizeraNeto, and T. Bastos-Filho, "A Low-Cost Lower-Limb Brain-Machine Interface Triggered by Pedaling Motor Imagery for Post-Stroke Patients Rehabilitation," IEEE Transactions on Neural Systems and Rehabilitation Engineering, vol. 28, no. 4, pp. 988-996, April 2020, doi: 10.1109/tnsre.2020.2974056.

[15] K. S. Mistry, P. Pelayo, D. G. Anil, and K. George, "An SSVEP based brain computer interface system to control electric wheelchairs," 2018 IEEE International Instrumentation and Measurement Technology Conference (I2MTC), Houston, TX, 2018, doi: 10.1109/ i2mtc.2018.8409632.

[16] Y. Y. Jang, T. H. Kim, and B. H. Lee, "Effects of Brain-Computer Interface-controlled Functional Electrical Stimulation Training on Shoulder Subluxation for Patients with Stroke: A Randomized Controlled Trial," Occup. Ther. Int., vol. 23, no. 2, pp. 175-185, Jun. 2016, doi: 10.1002/oti.1422.

[17] S. P. Zavala, J. L. L. Bayas, A. Ulloa, J. Sulca, J. L. M. López, and S. G. Yoo, "Brain Computer Interface Application for People with Movement Disabilities," International Conference on Human Centered Computing, vol.11354, pp. 35-47, Mar. 2019, doi: https://doi.org/10.1007/978-3-030-15127-0_4

[18] M. D. Novitasari, A. D. Wibawa, M. H. Purnomo, W. R. Islamiyah, and A. Fatoni, "Investigating EEG Pattern During DesignedHand Movement Tasks in Stroke Patients," 2020 International Seminar on Intelligent Technology and Its Applications (ISITIA), Surabaya, Indonesia, pp. 141-147, 2020, doi: 10.1109/isitia49792.2020.9163680.

[19] A. Guerra, D. Caceres, F. Merchan, and K.-H. Jo, "Real Time Operator Focus Monitoring System Based on a Brain-Computer Interface," 2020 IEEE 29th International Symposium on Industrial Electronics (ISIE), Delft, Netherlands, pp. 1415-1420, 2020, doi: 10.1109/isie45063.2020.9152264.
[20] S. M. Monowara, M. A. Shariar, M. S. U. Islam, and M. J. N. Jawad, "IoT Based Brain-Wave Assistive System for Paralyzed Individuals", B.Sc. Thesis, Electrical and Electronic Engineering, Brac University, Dec. 2020.

[21] P. G. Vinoj, S. Jacob, V. G. Menon, S. Rajesh, and M. R. Khosravi, "Brain-Controlled Adaptive Lower Limb Exoskeleton for Rehabilitation of Post-Stroke Paralyzed," IEEE Access, vol. 7, pp. 132628-132648, 2019, doi: 10.1109/access.2019.2921375.

\section{AUTHORS}

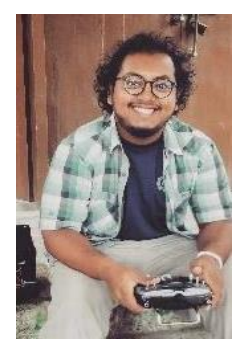

Md Ahnaf Shariar is currently pursuing his degree of Bachelor of Science in Electrical and Electronic Engineering, BRAC University, Bangladesh. He is currently working as an intern as a software and architectural visualization developer in a software company. $\mathrm{He}$ is interested in robotics and has participated in numerous robotics competition and secured several prizes in those competitions. He had been in the position of General Secretary of the Robotics club of Brac University.

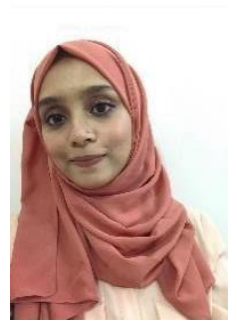

Syeda Maliha Monowara is currently pursuing her degree of Bachelors of Science in Electrical and Electronic Engineering, BRAC University, Bangladesh. She is interested in the research of robotics, neural networks and, neuroscience. She also aspires to work towards the betterment of humanity through applications of engineering.

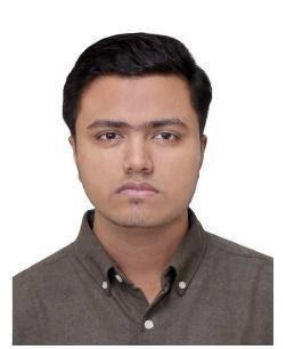

MD. Shafayat UI Islam as of late finished his graduation with a Bachelor of Science from BRAC University, Bangladesh. $\mathrm{He}$ is at present filling in as a data analyst in an outsourcing company. He is keen to work in the field of robotics and technology management. 


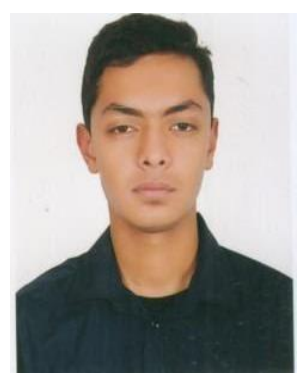

Muhammed Junaid Noor Jawad is a recent graduate of BRAC University, Bangladesh with a Bachelor of Science degree in Electrical and Electronic Engineering. His current scientific interest include artificial intelligence, neural networks and intelligent robots.

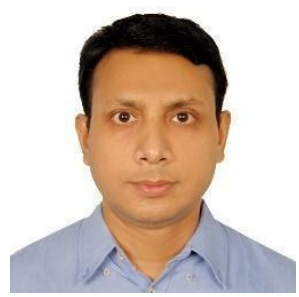

Saifur Rahman Sabuj was born in Bangladesh. He is currently working with the Electronics and Control Engineering Department as a Postdoctoral Research Fellow at Hanbat National University,
South Korea. He has been in the position of an assistant professor in the department of Electrical and Electronic Engineering at BRAC University, Bangladesh, since September 2017. From 2008 to 2013, he was a faculty member of Green University of Bangladesh, Metropolitan University, Sylhet and Bangladesh University. He received a B.Sc in Electrical, Electronic and Communication Engineering from Dhaka University, Bangladesh in 2007, an M.Sc at Engineering in the Institute of Information and Communication Technology, Bangladesh University of Engineering and Technology, Bangladesh in 2011, and a Ph.D. degree from the Graduate School of Engineering, Kochi University of Technology, Japan in 2017. His research interests include MIMO-OFDM/NOMA, Cooperative Communication, Cognitive Radio, Internet-of-things, Unmanned Aerial Vehicle and machine-to-machine for wireless communications. 